\title{
REMARKS ON THE ENTROPY AND HARNACK ESTIMATES FOR THE GAUSS CURVATURE FLOW
}

\author{
RICHARD S. HAMILTON
}

Consider a smooth compact strictly convex body $M^{n} \subseteq \mathbb{R}^{n+1}$ subject to wear under impact at a random angle, such as a stone being tossed on a beach. The probability of impact at any point $P$ is proportional to the Gauss curvature $K$. Thus the surface evolves in time by the Gauss curvature flow

$$
\frac{\partial P}{\partial t}=-K N
$$

where $N$ is the unit outward normal. This equation was first studied by Firey [Fi]. Later Tso $[\mathrm{Ts}]$ showed that the solution exists and stays smooth and strictly convex until $t \rightarrow T$ for some time $T$ when the diameter $L$ shrinks to zero. Thus we can assume $M^{n}$ shrinks to the origin 0. Recently Chow [Ch] proved an entropy and a Harnack estimate for this flow. We use these results to derive two useful corollaries.

Main Theorem 1. The diameter $L$ satisfies a dilation-invariant bound

$$
L \leq C(T-t)^{1 /(n+1)}
$$

This follows by combining Chow's upper bound on the entropy with a lower bound in terms of the least shadow area.

Main Theorem 2. The Gauss curvature $K$ satisfies a dilation-invariant bound

$$
K \leq\left(\frac{T}{t}\right)^{\frac{n}{n+1}} \frac{S}{T-t}
$$

where $S=\langle P, N\rangle$ is the support function around the limit point 0 . This result follow immediately from considering the Harnack estimate along radial lines. Combining these gives us the following. 
Corollary. The Gauss curvature flow satisfies a dilation-invariant bound

$$
K \leq C /(T-t)^{n /(n+1)} .
$$

This follows since $S \leq L$ everywhere.

Our appreciation to Ben Chow and Mike Gage for many useful conversations.

In this section we study the entropy integral

$$
E=\int K \ell n K d a_{\mathrm{M}}
$$

where $d a_{\mathrm{M}}$ is the area element on the surface $M$.

1a. In his important paper [Ch], Ben Chow proves the following result. Let $E_{t}$ be the value of the entropy at time $t, E_{0}$ its initial value, and $\sigma_{n}$ the area of the unit $n$-sphere.

1a1 Theorem. (Chow). The entropy has an upper bound

$$
E_{t} \leq E_{0}+\sigma_{n} \ell n(T / T-t)^{n /(n+1)} .
$$

Proof. See [Ch] p. 481.

1b. Now we give a useful lower bound on the entropy of any compact convex smooth hypersurface $M^{n}$ in $\mathbb{R}^{n+1}$.

1b1 Definition. The least shadow area $A_{\#}$ of $M$ is the least area of the image of $M$ under any projection onto a hyperplane $\mathbb{R}^{n}$ in $\mathbb{R}^{n+1}$.

Again we let $\sigma_{n}$ be the area of the unit $n$-sphere $S^{n}$, and we define the constant $C_{n}<\infty$ by

$$
C_{n}=\int_{S_{+}^{n}} \ln \sec \psi d a_{\mathrm{s}}
$$

where $S_{+}^{n}$ is the unit northern hemisphere, $\psi$ is the angle of declination from the north pole, and $d a_{\mathrm{s}}$ is the area element on the sphere. The improper integral is finite because $\sec \psi$ is inversely proportional to the distance from the equator, and the logarithm grows quite slowly. 
$1 \mathrm{b2}$ Theorem. The entropy $E$ has lower bound in terms of the least shadow area $A_{\#}$ given by

$$
E \geq \sigma_{n} \ell n\left(\sigma_{n} / 2 A_{\#}\right)-2 C_{n}
$$

Proof. Let $P$ be the projection whose shadow has the least area $A_{\#}$. The surface $M^{n}$ divides into two regions $B^{\prime}$ and $B^{\prime \prime}$ such that the projection $P$ is one-to-one on each region with image $A_{\#}$, while the orientation of the projection is opposite on their interiors. We then apply the following Lemma on both $B^{\prime}$ and $B^{\prime \prime}$ and add. Note that the images of $B^{\prime}$ and $B^{\prime \prime}$ in the Gauss sphere are two hemispheres of area $\sigma_{n} / 2$.

$1 \mathrm{b3}$ Lemma. Let $P$ be any projection of a convex surface $M^{n}$ on a hyperplane $R^{n}$ in $R^{n+1}$, let $B$ be any subset of $M^{n}$ with unit normal $N$ lying in one hemisphere, let $A_{P}(B)$ be the area of the image of $B$ under the projection $P$, and let $A_{s}(B)$ be the area of the image of $B$ in the Gauss sphere under the Gauss map $G$. Then the entropy of $B$

$$
E(B)=\int_{B} K \ln K d a_{M}
$$

has a lower bound

$$
E(B) \geq A_{S}(B) \ln \left[A_{s}(B) / A_{P}(B)\right]-C_{n} .
$$

Proof. Let $d a_{\mathrm{s}}, d a_{\mathrm{M}}$, and $d a_{\mathrm{P}}$ denote the area elements on the Gauss sphere $S^{n}$, the surface $M^{n}$ and the plane $R^{n}$ which is the image of the projection $P$. Rotate so the projection is vertical onto the horizontal hyperplane $R^{n}$, with the normal $N$ on $B$ always in the northern hemisphere $S_{+}^{n}$, and let $\psi$ be the angle of declination from the north pole. Then

$$
d a_{\mathrm{s}}=K d a_{\mathrm{M}} \quad \text { and } \quad d a_{\mathrm{M}}=\sec \psi d a_{\mathrm{P}} .
$$

This makes

$$
\int_{G(B)} 1 d a_{\mathrm{s}}=A_{\mathrm{s}}(B) \text { and } \int_{G(B)} \frac{1}{K \sec \psi} d a_{\mathrm{s}}=A_{\mathrm{P}}(B)
$$

while

$$
\int_{G(B)} \ln \left(\frac{1}{K \sec \psi}\right) d a_{\mathrm{s}}=-E(B)-C_{\mathrm{M}}
$$


Now Jensen's inequality tells us that the average value of the logarithm is less then or equal to the logarithm of the average, since the logarithm is concave. Hence

$$
\int_{G(B)} \ln \left(\frac{1}{K \sec \psi}\right) \frac{d a_{\mathrm{s}}}{\int_{G(B)} 1 d a_{\mathrm{s}}} \leq \ln \left(\int_{G(B)} \frac{1}{K \sec \psi} \frac{d a_{\mathrm{s}}}{\int_{G(B)} 1 d a_{\mathrm{s}}}\right)
$$

and the result follows.

1c. If we combine the upper bound and the lower bound on the entropy we get a lower bound on the least shadow area $A_{\#}$.

1c1 Theorem. The least shadow area $A_{\#}$ satisfies

$$
A_{\#}(t) \geq C_{\#}(T-t)^{n /(n+1)}
$$

where the constant $C_{\#}$ is given by

$$
C_{\#}=\frac{\sigma_{n}}{2 T^{n /(n+1)}} e^{-\left(E_{0}+2 C_{n}\right) / \sigma_{n}}
$$

Proof. We have

$$
\sigma_{n} \ell n\left(\sigma_{n} / 2 A_{\#}\right)-2 C_{n} \leq E \leq E_{0}+\sigma_{n} \ell n(T / T-t)^{n / n+1}
$$

and the result follows if we solve for $A_{\#}$.

The bound on the least shadow area gives us a bound on the diameter.

1d1 Definition. The diameter $L$ of a compact convex body $M$ is the length of the longest line segment contained in $M$. The volume $V$ of $M$ is the volume of the region enclosed.

1d2 Lemma. The volume $V$ in the Gauss curvature flow is given by

$$
V=\sigma_{n}(T-t) .
$$

Proof. We have

$$
\frac{d V}{d t}=-\int_{M} K d a_{\mathrm{M}}=-\sigma_{n}
$$

and $V \rightarrow 0$ as $t \rightarrow T$. 
$1 \mathrm{~d} 3$ Theorem. The diameter $L$ in the Gauss curvature flow satisfies dilationinvariant bounds

$$
c(T-t)^{1 /(n+1)} \leq L \leq C(T-t)^{1 /(n+1)}
$$

for some constants $c>0$ and $C<\infty$ which depend only on $n, T$ and $E_{0}$.

The proof depends on a geometric Lemma, which we give first.

1d4 Lemma. For any convex body $M^{n}$, the volume $V$, diameter $L$ and least shadow area $A_{\#}$ are related by

$$
V \geq \frac{1}{3} L A_{\#} .
$$

Proof. Find a line segment in $M^{n}$ with length $L$, let $P$ be the projection orthogonal to it, and let $A_{D}$ be the area of the shadow, the projection of $M^{n}$ under $P$ onto a hyperplane. Rotate so the projection $P$ is vertical onto a horizontal hyperplane $\mathbb{R}^{n}$, and translate so the line of length $L$ lies over the origin 0 in $\mathbb{R}^{n}$.

Introduce cylindrical coordinates $r, \theta$ and $z$, where $r$ is the distance from the origin in $\mathbb{R}^{n}, \theta \in S^{n-1}$ the angle, and $z$ the height in the perpendicular direction. The shadow of $M^{n}$ under $P$ will be a set given by an inequality

$$
0 \leq r \leq f(\theta)
$$

for some function $f(\theta)$, and the set $M^{n}$ itself will be the graph over this set of two functions $h_{1}(r, \theta)$ and $h_{2}(r, \theta)$, while the region inside is given by

$$
h_{1}(r, \theta) \leq z \leq h_{2}(r, \theta) .
$$

Note that when $r=f(\theta)$ we have

$$
h_{1}(f(\theta), \theta)=h_{2}(f(\theta), \theta)=h(\theta)
$$

for some function $h(\theta)$. If we translate in the $z$-direction so that the line segment of length $L$ starts at the origin in $R^{n+1}$, we get

$$
h_{1}(0, \theta)=0 \quad \text { and } \quad h_{2}(0, \theta)=L
$$

for all $\theta$. 
Since $h_{1}$ is concave and $h_{2}$ is convex, we must have

$$
h_{1}(r, \theta) \leq \frac{r}{f(\theta)} h(\theta)
$$

and

$$
h_{2}(r, \theta) \geq \frac{r}{f(\theta)} h(\theta)+\left[1-\frac{r}{f(\theta)}\right] L .
$$

The volume $V$ inside $M^{n}$ is given by

$$
V=\int_{S^{n-1}} \int_{r=0}^{f(\theta)} \int_{z=h_{1}(r, \theta)}^{h_{2}(r, \theta)} r d z d r d \theta
$$

in cylindrical coordinates. Using the above inequalities and doing the two inner integrals gives

$$
V \geq \frac{L}{6} \int_{S^{n-1}} f(\theta)^{2} d \theta .
$$

On the other hand, the area of the shadow is given by

$$
A_{b}=\int_{S^{n-1}} \int_{r=0}^{f(\theta)} r d r d \theta
$$

in polar coordinates, so

$$
A_{b}=\frac{1}{2} \int_{S^{n-1}} f(\theta)^{2} d \theta
$$

which makes

$$
V \geq \frac{1}{3} L A_{b}
$$

Since $A_{\#}$ is the least shadow area, $A_{b} \geq A_{\#}$. This proves the Lemma.

Now we can finish the proof of Theorem $1 \mathrm{~d} 3$. We have

$$
L \leq 3 V / A_{\#}
$$

by the previous Lemma,

$$
V=\sigma_{n}(T-t)
$$

by Lemma $1 \mathrm{~d} 2$, and

$$
A_{\#} \geq C_{\#}(T-t)^{n /(n+1)}
$$

by Theorem 1c1. This gives

$$
L \leq C(T-t)^{1 /(n+1)}
$$


for a constant $C$ determined by $C_{\#}$ and $n$. Finally, $M^{n}$ must be contained in a ball of radius $L$, so

$$
V \leq \sigma_{n} L^{n+1}
$$

which gives a lower bound on $L$ of the form

$$
L \geq c(T-t)^{1 /(n+1)}
$$

where $c$ depends only on $n$.

2.

In this section we study the Harnack inequality for the Gauss curvature flow. It tells us that if the Gauss curvature $K$ is large at some point after some time has elapsed, it must be comparably large at nearby points after some more time has elapsed. This is a common feature in heat equations where some function is always positive, as $K$ is in this case.

2a. The Harnack estimate for the Gauss curvature flow was derived by Chow in [Ch]. He proves the following result (in his Theorem 3.7).

2a1 Theorem. (Chow). For any points $X_{1}$ on $M^{n}$ at time $t_{1}$ and $X_{2}$ on $M^{n}$ at time $t_{2}$ with $0<t_{1}<t_{2}$ we have

$$
\frac{K\left(X_{2}, t_{2}\right)}{K\left(X_{1}, t_{1}\right)} \geq\left(\frac{t_{1}}{t_{2}}\right)^{\frac{n}{n+1}} e^{-\theta / 4}
$$

where $\theta$ is the integral over any path $X=X(t)$ on the surface at each time $t$ with $X=X_{1}$ at $t=t_{1}$ and $X=X_{2}$ at $t=t_{2}$ given by

$$
\theta=\int_{t_{1}}^{t_{2}} K H_{i j}^{-1} V^{i} V^{j} d t
$$

where $H_{i j}^{-1}$ is the inverse of the second fundamental form and $V^{i}$ is the tangential component of the velocity of the path $X$, so that

$$
\frac{d X^{\alpha}}{d t}=-K N^{\alpha}+V^{i} D_{i} X^{\alpha}
$$

in local coordinates on $M^{n}$.

Chow obtains this result by integrating the following differential Harnack inequality over the path. 
2a2 Theorem. (Chow). In any solution to the Gauss curvature flow for $t>0$, we have

$$
\frac{\partial K}{\partial t} \geq H_{i j}^{-1} D_{i} K D_{j} K-\frac{n}{(n+1) t} K
$$

Proof. See [Ch], p.478 and multiply by $K$.

2b. A very useful version of the Harnack estimate can be obtained by integrating over a path in $\mathbb{R}^{n+1}$ which is just a straight line segment. Of course the velocity will not be constant, but must be adjusted to stay on the surface $M^{n}$ at each time $t$. This requires that the line segment from beginning to end must always be transversal to the surface $M^{n}$.

2b1 Theorem. Along any straight line segment transversal to $M^{n}$ the function

$$
t^{n /(n+1)} K \sec \theta
$$

always increases, where $\theta$ is the angle between the line segment and the normal to the surface.

Proof. Suppose the line segment has a unit velocity vector $U$. Then

$$
\frac{d X^{\alpha}}{d t}=\sigma U^{\alpha}
$$

where $\sigma$ must be adjusted to stay on the surface. Dividing $d X / d t$ into tangential and normal components

$$
\frac{d X^{\alpha}}{d t}=-K N^{\alpha}+V^{i} D_{i} X^{\alpha}
$$

as before. Since $N \cdot U=-\cos \theta$, we need to take

$$
\sigma=K \sec \theta \quad \text { and } \quad V_{i}=U^{\alpha} \cdot D_{i} X^{\alpha}
$$

as we see by dotting with $U$ or $D_{j} X^{\alpha}$.

The space and time derivatives of the unit outward normal $N$ are given by Chow in $[\mathrm{Ch}]$ as

$$
D_{i} N^{\alpha}=H_{i j} D_{j} X^{\alpha}
$$

and

$$
\frac{\partial N^{\alpha}}{\partial t}=D_{i} K D_{i} X^{\alpha}
$$


Now along the line segment

$$
\frac{d N^{\alpha}}{d t}=\frac{\partial N^{\alpha}}{\partial t}+V_{i} D_{i} N^{\alpha}
$$

so

$$
\frac{d N^{\alpha}}{d t}=D_{i} K D_{i} X^{\alpha}+H_{i j} V_{i} D_{j} X^{\alpha}
$$

Then

$$
\frac{d N}{d t} \cdot \frac{d X}{d t}=V_{i} D_{i} K+H_{i j} V_{i} V_{j} .
$$

Now since $\sigma=K \sec \theta$,

$$
N \cdot U=-\cos \theta=-K / \sigma
$$

and

$$
\frac{d^{2} X}{d t^{2}}=\frac{d \sigma}{d t} U
$$

so

$$
N \cdot \frac{d^{2} X}{d t^{2}}=-\frac{K}{\sigma} \frac{d \sigma}{d t}=-K \frac{d}{d t} \ln (K \sec \theta) .
$$

Also

$$
N \cdot \frac{d X}{d t}=-K
$$

so

$$
\frac{d}{d t}\left(N \cdot \frac{d X}{d t}\right)=-\frac{d K}{d t}
$$

differentiating along the path, where

$$
\frac{d K}{d t}=\frac{\partial K}{\partial t}+V_{i} D_{i} K \text {. }
$$

Finally, note that

$$
\frac{d}{d t}\left(N \cdot \frac{d X}{d t}\right)=N \cdot \frac{d^{2} X}{d t^{2}}+\frac{d N}{d t} \cdot \frac{d X}{d t} .
$$

Combining these results gives

$$
\frac{\partial K}{\partial t}+2 V_{i} D_{i} K+H_{i j} V_{i} V_{j}=K \frac{d}{d t} \ln (K \sec \theta)
$$

along the straight line segment.

Now Chow's differential Harnack inequality (Theorem 2a2) is equivalent to the statement that

$$
\frac{\partial K}{\partial t}+2 V_{i} D_{i} K+H_{i j} V_{i} V_{j} \geq-\frac{n}{(n+1) t} K
$$


for any vector $V_{i}$, as the worst possible choice of $V_{i}$ is

$$
V_{i}=-H_{i j}^{-1} D_{j} K
$$

and even this works. Therefore

$$
K \frac{d}{d t} \ln (K \sec \theta) \geq-\frac{n}{(n+1) t} K
$$

or equivalently

$$
\frac{d}{d t} \ln \left(t^{n /(n+1)} K \sec \theta\right) \geq 0
$$

which shows $t^{n /(n+1)} K \sec \theta$ increases along the line segment, as desired.

2c. We can apply this result to radial paths out of the limit point 0 , since they must all be transversal to the flow.

2c1 Theorem. The Gauss curvature satisfies the bound

$$
K \leq\left(\frac{T}{t}\right)^{\frac{n}{n+1}} \frac{S}{T-t}
$$

where $S=\langle P, W\rangle$ is the support function around the limit point 0 .

Proof. The angle $\theta$ between the radial line and the unit normal $N$ is given by

$$
S=\langle P, W\rangle=r \cos \theta
$$

where $r=|P|$ is the distance to 0 . Now along the radial line

$$
\frac{d r}{d t}=-K \sec \theta
$$

and we know

$$
t^{n(n+1)} K \sec \theta
$$

is increasing. Thus for $t_{0} \leq t<T$ we get

$$
t^{n /(n+1)} K \sec \theta \geq t_{0}^{n /(n+1)} K_{0} \sec \theta_{0}
$$

and using $t<T$ and

$$
\sec \theta_{0}=r_{0} / S_{0}
$$

we get

Now

$$
r_{0}\left(t_{0} / T\right)^{n /(n+1)} K_{0} / S_{0} \leq-\frac{d r}{d t} .
$$

$$
\int_{t_{0}}^{T}-\left(\frac{d r}{d t}\right) d t=r_{0}
$$


so we have

$$
r_{0}\left(t_{0} / T\right)^{n /(n+1)}\left(T-t_{0}\right) K_{0} / S_{0} \leq r_{0}
$$

after integrating, or

$$
K_{0} \leq\left(\frac{T}{t_{0}}\right)^{\frac{n}{n+1}} \frac{S}{T-t_{0}}
$$

which proves the Theorem if we replace $t_{0}$ by $t$.

\section{REFERENCES}

[Ch] Chow, B., On Harnack's inequality and entropy for the Gaussian Curvature Flow, Comm. Pure Appl. Math. XLIV (1991), 469-483.

[F] Firey, W., Shapes of worm stones, Mathematica 21 (1974), 1-11.

[Ts] Tso, K., Deforming a hypersurface by its Gauss-Kronecker curvature, Comm. Pure Appl. Math. 38 (1985), 867-882.

University of California, SAn Diego, U. S. A.

RECEIVED SePtEMBER 27, 1993 